\title{
Overexpression of PGA37/MYB118 and MYB115 promotes vegetative-to-embryonic transition in Arabidopsis
}

\author{
Xingchun Wang ${ }^{1,2, *}$, Qi-Wen $\mathrm{Niu}^{3, *}$, Chong Teng ${ }^{1,2}$, Chao $^{1, i^{1,2}}$, Jinye $\mathrm{Mu}^{1,2}$, Nam-Hai Chua ${ }^{3}$, Jianru Zuo \\ ${ }^{I}$ State Key Laboratory of Plant Genomics and National Plant Gene Research Center (Beijing), Institute of Genetics and Develop- \\ mental Biology, Chinese Academy of Sciences, Beijing 100101, China; ${ }^{2}$ Graduate School, Chinese Academy of Sciences, Beijing \\ 100049, China; ${ }^{3}$ Laboratory of Plant Molecular Biology, The Rockefeller University, New York, NY 10021, USA
}

Formation of somatic embryos from non-germline cells is unique to higher plants and can be manipulated in a variety of species. Previous studies revealed that overexpression of several Arabidopsis genes, including WUSCHEL (WUS)/PLANT GROWTH ACTIVATOR6 (PGA6), BABY BOOM, LEAFY COTYLEDON1 (LEC1), and LEC2, is able to cause vegetative-to-embryonic transition or the formation of somatic embryos. Here, we report that a gain-offunction mutation in the Arabidopsis PGA37 gene, encoding the MYB118 transcription factor, induced vegetative-toembryonic transition, the formation of somatic embryos from root explants, and an elevated $L E C 1$ expression level. Double mutant analysis showed that WUS was not required for induction of somatic embryos by PGA37/MYB118. In addition, overexpression of $M Y B 115$, a homolog of $P G A 37 / M Y B 118$, caused a pga37-like phenotype. A myb118 myb115 double mutant did not show apparent developmental abnormalities. Collectively, these results suggest that PGA37/ $M Y B 118$ and MYB115 promote vegetative-to-embryonic transition, through a signaling pathway independent of WUS.

Keywords: Arabidopsis, embryogenic competence, PGA37/MYB118, MYB115, somatic embryos Cell Research (2009) 19:224-235. doi: 10.1038/cr.2008.276; published online 12 August 2008

\section{Introduction}

In higher plants, life cycle starts from the fertilization of an egg cell by a sperm to produce a zygotic embryo. After fertilization, a zygotic embryo develops into a mature embryo through a series of developmental processes referred to as embryogenesis. In addition to the zygotic embryogenesis pathway, embryos can also arise from somatic tissues without undergoing meiosis and fertilization. Asexual embryos can be produced by manipulating somatic or gametophytic cells from a variety of species $[1,2]$. Moreover, the process of somatic embryo development is similar to that of zygotic embryos which are usually embedded inside maternal tissues, making them inaccessible to analysis. Thus, somatic embryos provide

*These two authors contributed equally to this work.

Correspondence: Jianru Zuo

Fax: +86-10-64873428

E-mail: jrzuo@genetics.ac.cn

Received 27 June 2008; revised 30 June 2008; accepted 30 June 2008; published online 12 August 2008 an attractive model for studying embryogenesis.

In vitro induction of somatic embryo formation is initiated by inducing de-differentiation of somatic cells, usually requiring high concentrations of 2,4-dichlorophenoxyacetic acid (2,4-D). Subsequently, the de-differentiated cells or calli re-differentiate and develop as somatic embryos by an unknown mechanism. Whereas phytohormones are generally believed to activate a signaling pathway for the induction of embryogenic competence, a number of genetic loci have also been shown to play critical roles during somatic embryo development [24]. Mutations in the Arabidopsis LEAFY COTYLEDON1 ( $L E C 1)$ and $L E C 2$ genes cause similar defects in embryo maturation and partially transform cotyledons into leaves $[5,6]$. LEC1 and LEC2 encode a CCAAT-binding factortype transcription factor and a plant-specific B3 transcription factor, respectively [7, 8]. Ectopic expression of these two embryo-specific genes causes the formation of somatic embryos on vegetative tissues $[4,7,8]$. A recent study shows that the auxin biosynthetic genes $Y U C 2$ and $Y U C 4$ are rapidly induced by $L E C 2$, and YUC4 is likely a direct target of $L E C 2$ [4]. These observations establish 
a molecular link between the regulation of embryogenic competence and the auxin activity. On the other hand, whereas $L E C 1$ and $L E C 2$ are key regulators controlling embryonic identity, the Arabidopsis PICKLE (PKL) gene, encoding a CHD3 chromatin-remodeling factor, appears to play an opposite role. The $p k l$ mutant displays some embryonic traits after germination, including a higher capacity of embryogenic competence, the production of somatic embryos from root explants, and an elevated LEC1 expression [9-11]. Because CHD3 proteins are involved in transcriptional repression, $P K L$ has been proposed to prevent re-expression of the embryonic developmental state during germination, likely through the regulation of gibberellin (GA) signaling $[10,11]$. A more recent study identified three functionally redundant $V P 1 /$ ABI3-LIKE (VAL) genes encoding B3 domain-containing proteins [12]. Whereas vall shows a $p k l$-like phenotype, the vall val2 double mutant produces embryo-like structures around root and apical meristem regions, which is associated with increased expression of $L E C 1, L 1 L$, $A B I 3$, and FUSCA3 (FUS3). It has been proposed that VAL factors may function as transcriptional repressors of the $L E C 1 / B 3$ genes in conjunction with PKL or related factors [12].

Using reverse genetic approaches, several genes have been shown to have the capacity to induce the formation of embryonic calli or somatic embryos from vegetative tissues. The carrot SOMATIC EMBRYOGENESIS RECEPTOR-LIKE KINASE (SERK) gene was shown to mark vegetative-to-embryonic transition in carrot suspension cell cultures [13], and its Arabidopsis orthologous gene was found to enhance embryogenic competence in cell cultures as well [14]. The BABY BOOM (BBM) gene, encoding an AP2/ERF-type transcription factor, was identified from Brassica napus and Arabidopsis thaliana. Ectopic expression of $B B M$ results in the formation of somatic embryos and cotyledon-like structures on seedlings [15]. Moreover, overexpression of the Arabidopsis AGAMOUS-Like 15 (AGL15) gene and its homologous gene in soybean, which encode MADS domaincontaining proteins, enhances the formation of secondary embryos and somatic embryos [16, 17]. The promotion of somatic embryo formation by $A G L 15$ is attributed, at least in part, to a reduced GA level [18]. This notion is consistent with the observation made in the $p k l$ mutant that displays a GA-deficiency phenotype $[9,10]$, and the observation that FUS3, encoding a $\mathrm{B} 3$ transcription factor critical for embryogenesis, negatively regulates GA biosynthesis [19].

We have used a functional screening strategy to identify Arabidopsis gain-of-function mutants, plant growth activator (pga), by culturing T-DNA-transformed root explants on a medium containing auxin but without cytokinin. In this screen, a chemical-inducible activation tagging vector was used, and therefore the expression of the mutant phenotype was strictly dependent on the chemical inducer estradiol $[20,21]$. Mutants identified from this screen were presumed to conditionally overexpress key components that regulate somatic embryo formation or the cytokinin response [21, 22]. The pga6 mutant was found to efficiently produce somatic embryos on seedlings and explants in the presence of the inducer. Molecular analysis demonstrated that PGA6 was identical to the homeobox gene WUSCHEL (WUS) [21], which regulates stem cell fate in shoot and floral meristems [23-25]. Moreover, ectopic expression of WUS also induces shoot and floral stem cell activities in roots and stems, respectively [26, 27]. These observations suggest that WUS/PGA6 plays a critical role in the maintenance of the identity of embryonic stem cells. Here, we report the characterization of the pga37 mutant, which displays a similar but distinctive phenotype compared to pga6. The $P G A 37$ gene, encoding a MYB transcription factor, induces embryogenic competence in a $W U S$-independent manner.

\section{Results}

\section{The pga37 mutant phenotype}

In previous genetic screens for pga mutants, we have identified two groups of mutants that displayed the ability to form either somatic embryos or shoots [21, 22]. For example, overexpression of the PGA6/WUS gene causes vegetative-to-embryonic transition [21]. The PGA22 gene, encoding isopentenyl transferase 8 (IPT8), catalyzes the first rate-limiting step of cytokinin biosynthesis, and the pga22 gain-of-function mutant shows a cytokinin overproduction phenotype, including the regeneration of shoots in the absence of externally supplied cytokinin [22].

Here, we report the characterization of pga37, which showed a phenotype similar to that of pga6. When cultured on the screening medium (SCM; containing IAA, kanamycin, and the chemical inducer $17-\beta$-estradiol but without cytokinins; see Materials and Methods) for 7-10 days, pga37 root explants produced green-yellowish embryonic calli (Figure 1A), which were not found on control medium without the chemical inducer estradiol (Figure 1B). Upon culturing for a longer period of 3-5 weeks, somatic embryos were generated from embryonic calli (Figure 1C and 1D). Scanning electron microscopic analysis revealed the presence of somatic embryos at a variety of developmental stages, including the globular, heart, and cotyledon stage (Figure 1E-1G). Similar to 

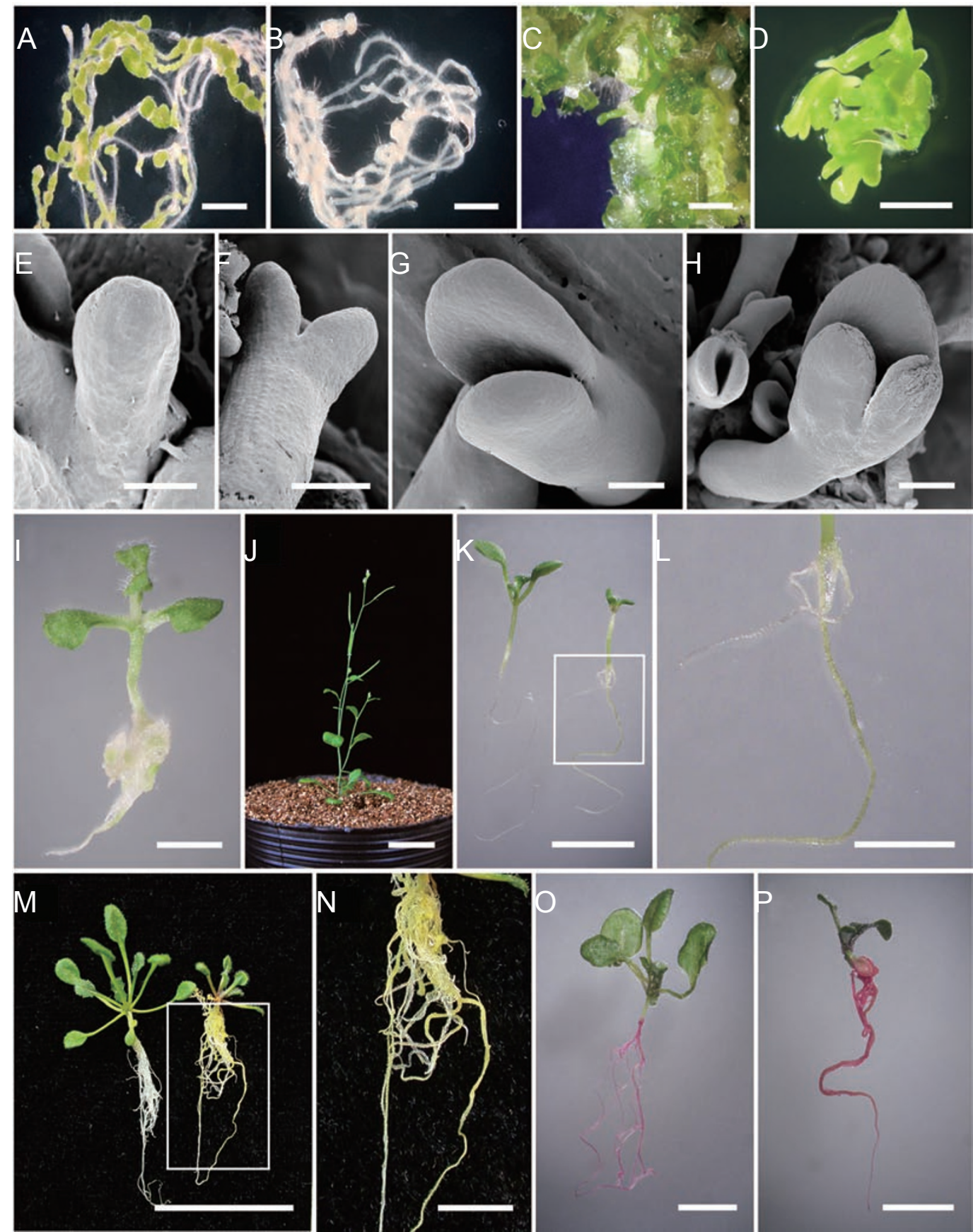

Figure 1 The pga37 gain-of-function mutant phenotype. (A) pga37 root explants cultured on SCM containing $10 \mu \mathrm{M}$ estradiol for 7 days. Note: yellowish green embryonic calli are visible. (B) pga37 root explants cultured on SCM without estradiol for 7 days. (C) pga37 root explants cultured on SCM containing $10 \mu \mathrm{M}$ estradiol for 5 weeks. Somatic embryos are formed on the explants. (D) An enlarged view of panel (C). (E-H) Scanning electron microscopy of somatic embryos at various developmental stages. Images were taken from pga37 root explants cultured on SCM for 3-5 weeks. (E) A somatic embryo at the globular stage. (F) A somatic embryo at the heart stage. (G) A somatic embryo at the cotyledon stage. (H) An abnormal somatic embryo with three cotyledons. (I) A pga37 seedling germinated from a somatic embryo. (J) A soil-grown pga37 seedling derived from a somatic embryo. (K) Seven-day-old pga37 seedlings germinated and grown on a MS medium in the absence (left) or presence (right) of $10 \mu \mathrm{M}$ estradiol. (L) An enlarged view of the boxed region in panel (K). (M) Three-week-old pga37 seedlings germinated and grown on a MS medium in the absence (left) or presence (right) of $10 \mu \mathrm{M}$ estradiol. (N) An enlarged view of the boxed region in panel (M). (O and $\mathrm{P}$ ) Fat Red 7B staining of 2-week-old pga37 seedlings germinated and grown on MS medium in the absence $(\mathrm{O})$ or presence $(\mathrm{P})$ of $10 \mu \mathrm{M}$ estradiol. Bar, $0.5 \mathrm{~mm}(\mathrm{~A}-\mathrm{D}), 50 \mu \mathrm{m}(\mathrm{E}), 100 \mu \mathrm{m}(\mathrm{F}-\mathrm{H}), 2 \mathrm{~mm}(\mathrm{I}$ and $\mathrm{L}), 5 \mathrm{~mm}(\mathrm{~K}, \mathrm{~N}-\mathrm{P})$, and $2 \mathrm{~cm}(\mathrm{~J}$ and $\mathrm{M})$. 
that observed in the pga6 mutant, somatic embryos with three cotyledons were occasionally observed (Figure $1 \mathrm{H})$.

After transfer from SCM to MS medium [28] without the inducer estradiol, these somatic embryos were able to continue development into healthy seedlings, and plantlets were fertile and set seeds (Figure 1I and 1J). When seeds harvested from pga37 plantlets were germinated and grown in the absence of the inducer, pga37 plants were indistinguishable from wild-type (WT) plants. However, when germinated and grown in the presence of inducer, pga37 seedlings were smaller than WT seedlings, and produced dark green cotyledons and true leaves (Figure $1 \mathrm{~K}$ and $1 \mathrm{M}$ ). Moreover, pga37 roots and the junction region between the root and the hypocotyl turned light green (Figure 1K-1N) similar to that previously observed in pickle ( $p k l)$ mutant roots $[9,10]$. Notably, the pga 37 mutation also caused the accumulation of storage compounds such as lipids, which were normally found in embryos and seeds, in roots and other vegetative tissues (Figure $1 \mathrm{O}$ and $1 \mathrm{P}$ ).

Taken together, these observations suggest that the pga37 mutation is capable of promoting vegetative-toembryonic transition, thus likely representing a key genetic locus regulating Arabidopsis embryogenesis.

\section{Molecular characterization of the PGA37 gene}

To determine the genetic basis of pga37 (in the Wassilewskija (WS) background), we crossed the mutant with WT (WS). All tested F1 progeny from the cross showed the mutant phenotype in an estradiol-dependent manner. In an F2 population obtained from self-pollinated F1 plants, the phenotype of forming somatic embryos on explants segregated in a 3:1 ratio (mutant: $\mathrm{WT}=$ $85: 26$ ). These results indicated that pga37 is a dominant mutation in a single nuclear gene, and its mutant phenotype was strictly dependent on the inducer. Because of the dependency on the inducer, the mutated gene should be tightly linked to a T-DNA insert carrying the $O^{\text {LexA }}-46$ promoter/enhancer. We identified the Arabidopsis genomic sequences flanking the $O^{\text {LexA }}-46$ promoter/ enhancer by inverse PCR [29]. A T-DNA was found to be inserted approximately 7.8 kilo base pairs $(\mathrm{kb})$ upstream from the putative translation start codon of At3g27785, which encodes the transcription factor MYB118. The left border (LB) of the T-DNA insertion was adjacent to the 5 '-end of MYB118, thus allowing the inducible $O^{\operatorname{LexA}}-46$ promoter/enhancer to activate the expression of the transcription factor gene (Figure 2A). Using an F2 population obtained from a cross between pga37 (WS) and WT (Columbia- 0 or Col-0), the mutation was roughly mapped onto chromosome III, approximately $4.4 \mathrm{cM}$ from the simple sequence length polymorphism maker ciw11 (45 F2 plants were tested). MYB118 is approximately $4.0 \mathrm{cM}$ south of ciw11 (Figure 2A). These results suggest that the pga37 mutation was likely caused by the identified T-DNA insertion.

In this $7.8-\mathrm{kb}$ region between the insertion site and MYB118, neither protein- nor putative small RNAencoding genes could be annotated using various prediction programs or by a manual search. Northern blot analysis showed that MYB 118 expression was inducible in the pga 37 mutant by estradiol in a dose-dependent manner (Figure 2B); moreover, the severity of the mutant phenotype was correlated with the MYB118 expression level (Figure 2B and 2C). A MYB 118 cDNA fragment spanning the entire coding sequences was cloned into the estradiol-inducible expression vector pER10 [20] and the resulting construct pER10-MYB118 was transformed into WT plants (WS). Expression of the MYB118 transgene was highly inducible by estradiol (Figure 2D). Upon induction, the pga37 mutant phenotype was recapitulated in pER10-MYB118 transgenic plants, including the somatic embryo formation phenotype (Figure 2E) and retarded growth and development of the transgenic seedlings (Figure 2F). Notably, when germinated under higher concentrations of inducer, growth and development of pER10-MYB 118 transgenic plants were completely arrested, and the treated plants produced calluslike tissues without any recognizable structures of roots and hypocotyls (Figure $2 \mathrm{~F}$ and $2 \mathrm{G}$ ). Note that multiple independent pER10-MYB118 transgenic lines were analyzed and similar results were obtained.

A stronger phenotype of the pER10-MYB118 transgenic plants compared to pga37 was most likely caused by higher transgene expression levels. In most transgenic lines, MYB118 expression was substantially higher than that in pga37, as shown in Figure 2D for a representative transgenic line. Moreover, because of the relative distal position of the T-DNA insertion from MYB118 in the pga37 genome (approximately $7.8 \mathrm{~kb}$ ), the $O^{\text {LexA }}-46$ sequence was unlikely to directly activate the MYB 118 transcription as a promoter, but rather it might act as a transcriptional enhancer. Consistent with this notion, only a single transcript was detected in inducer-treated pga37 plants, which was different from that observed in pga6 and pga22. In pga6 and pga22, two species of transcripts were detected, of which the shorter species presumably originated from the native promoters whereas the longer one likely initiated from the $O^{L e x A}-46$ promoter $[21,22]$. Nevertheless, the data presented above confirmed that overexpression of MYB 118 was sufficient to recapitulate the pga37 mutant phenotype. 
A

PGA37

(At3g27785)

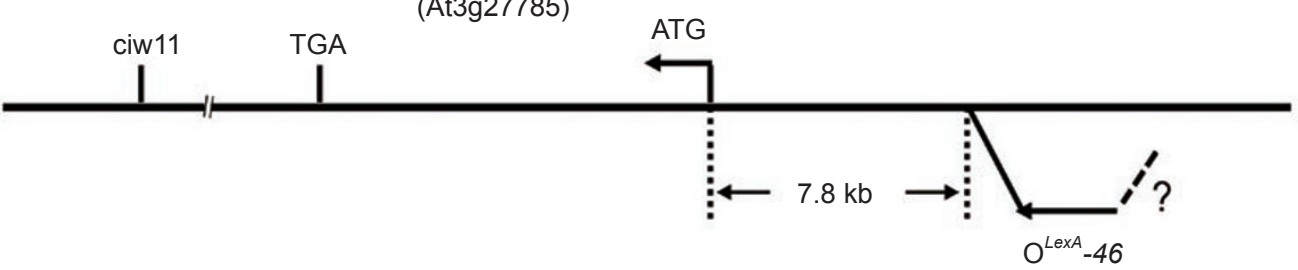

B

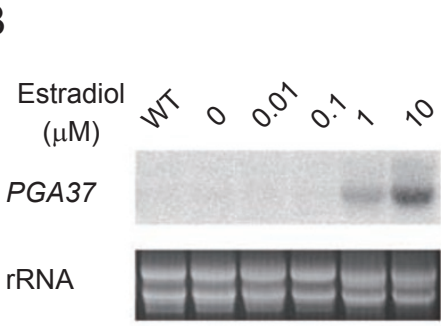

C
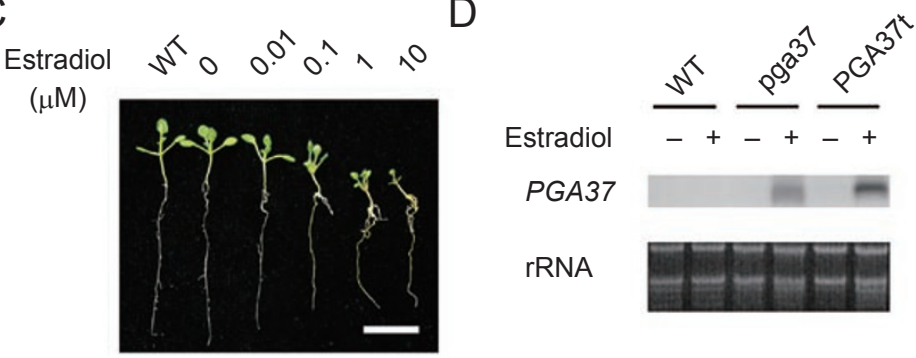

E

$\mathrm{F}$

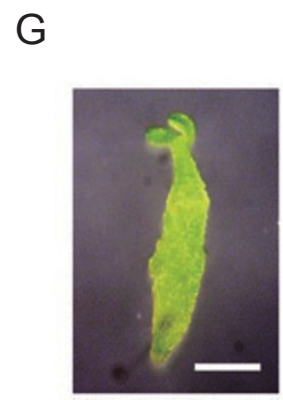

Figure 2 Molecular characterization of PGA37. (A) A schematic map of the pga37 mutant genome (not in scale). The insertion position is indicated by an arrow that also shows the orientation of left border (LB) of the T-DNA. The insertion position of the right border (RB) is unclear, as shown by a question mark. The pga37 mutation was mapped to approximately $4.4 \mathrm{~cm}$ from the ciw11 marker. (B) Expression of PGA37 in the pga37 mutant. Two-week-old pga37 seedlings were treated with different concentrations $(\mu \mathrm{M})$ of estradiol for $16 \mathrm{~h}$ as indicated on the top of the panel. Total RNA was analyzed by northern blot hybridization using a PGA37 cDNA fragment as a probe. WT: wild type (Wassilewskija or WS) seedlings treated with dimethyl sulfoxide (DMSO; a solvent for estradiol). (C) Dose-dependency of the pga37 mutant phenotype. Two-week-old pga37 seedlings germinated and grown on MS medium containing various concentrations of estradiol $(\mu \mathrm{M})$ as indicated on the top of the panel. WT: DMSO-treated WS seedlings. (D) Expression of PGA37 in pER10-PGA37 transgenic plants. Two-week-old seedlings of WT, pga37 and pER10-PGA37 transgenic plants (PGA37t) were treated with (+) or without (-) $10 \mu \mathrm{M}$ estradiol for $16 \mathrm{~h}$, and total RNA was analyzed by northern blot hybridization as described in panel (B). (E) Formation of somatic embryos on root explants of a pER10-PGA37 transgenic line. The explants were cultured on SCM for 5 weeks. (F) Phenotype of pER10$P G A 37$ transgenic plants. Two-week-old pER10-PGA37 seedlings germinated and grown on MS medium containing various concentrations of estradiol $(\mu \mathrm{M})$ as indicated on the top of the panel. WT: DMSO-treated WS seedlings. Note that the transgenic plants showed a stronger phenotype than pga37 (see panel (C) and text for details). (G) An enlarged view of a pER10PGA37 transgenic seedling in panel (F) marked by an arrow (grown in the presence of $10 \mu \mathrm{M}$ estradiol). No distinctive root and hypocotyl structures were found, and embryonic calli were formed on the plantlet. Bar, $1 \mathrm{~cm}(\mathrm{C}$ and $\mathrm{F})$ and $1 \mathrm{~mm}(E$ and $\mathrm{G})$.

A PGA37::GUS reporter gene is preferentially expressed in embryos and endosperm

A reverse transcription-PCR (RT-PCR) analysis detected $P G A 37$ expression in siliques but not in any other tissues/organs (Figure 3A). To more precisely follow the $P G A 37$ expression pattern, a $P G A 37$ promoter:: $\beta$ glucuronidase (PGA37::GUS) reporter gene was trans- formed into WT plants. A 2.5-kb Arabidopsis genomic DNA fragment, including the putative promoter sequence, 5'-untranslated region (UTR), and sequences encoding the first 12 amino acid residues of MYB118, was fused in frame to the GUS coding sequence. The $P G A 37:: G U S$ expression was specifically detected in developing embryos, starting approximately at the early 


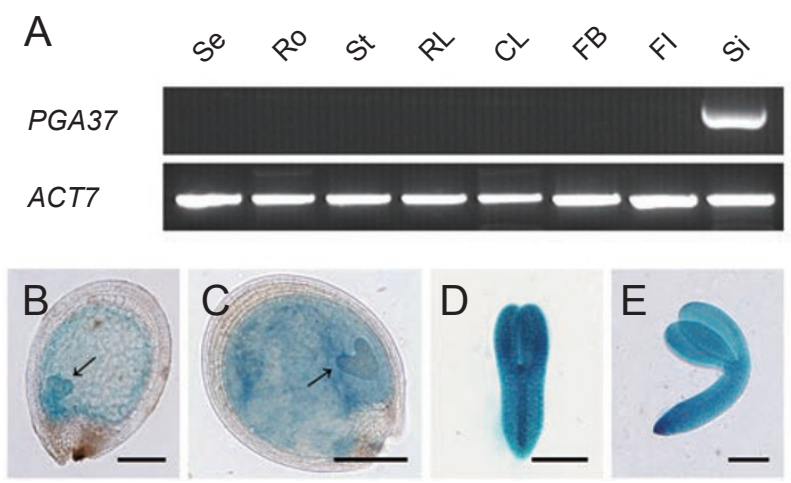

Figure 3 Expression pattern of PGA37. (A) Total RNA from various tissues/organs of WT plants was subjected to reverse transcription (RT)-PCR analysis. Two micrograms of RNA was used for RT using oligo-dT as a primer, and the PCR was cycled for 30 times. Primers used in PCR were located in exons 2 and 4 of $P G A 37$, respectively (see Figure $6 \mathrm{I}$ for the positions and orientation of the primers). Actin7 (ACT7; At5g09810) was used as an internal control. Se: seedling; Ro: root; St: stem; RL: rosette leaf; CL: cauline leaf; FB: flower bud; Fl: flower; Si: silique. (BE) Analysis of $\beta$-glucuronidase (GUS) activity in PGA37::GUS transgenic plants. At least five independent transgenic lines were analyzed and similar results were obtained. (B) Early heart stage; (C) late heart stage; (D) torpedo stage; (E) cotyledon stage. Bar $=100 \mu \mathrm{m}$.

heart stage and throughout embryogenesis (Figure 3B3E). GUS activity was restricted to endosperm and embryos, but not detectable in the seed coat (Figure 3B and $3 \mathrm{C})$. Upon entering the cotyledon stage, GUS expression was also detected in embryos (Figure 3D and 3E), but became undetectable after germination. Consistent with the results of RT-PCR analysis (Figure 3A), the $P G A 37:: G U S$ expression was not detected in any other tissues/organs at various developmental stages. The embryo- and endosperm-specific expression pattern of $P G A 37$ suggests that it plays a role during embryogenesis.

Increased expression of embryonic genes in pga37

Data presented above show that $P G A 37 / M Y B 118$ is capable of inducing vegetative-to-embryonic transition, leading to the formation of somatic embryos. To better understand the molecular mechanism underpinning this transition, we investigated the expression of several embryo- or seed-specific genes in pga37. Root explants derived from WT and pga37 plants were cultured on SCM for different times. In pga37 explants, green-yellowish embryonic calli were usually formed around 7-10 days, and somatic embryos were produced around 20-30 days. Expression of $L E C 1$ was detectable around day 1, whereas expression of LEC2, FUS3 and WUS became detect- able in later stages (Figure 4A). A similar expression pattern was also observed in explants derived from pER10$P G A 37$ transgenic plants. To confirm this result, pER10$P G A 37$ transgenic plants germinated and grown on MS medium were treated with inducer for varying times, and $L E C 1$ expression was analyzed by RT-PCR. LEC1 expression could be detected after $6 \mathrm{~h}$ of induction, and a higher expression level was found at later time points (Figure 4B). Under these assay conditions, expression of WUS, FUS3, and LEC2 was not detected (data not shown). These results suggest that $P G A 37$ is able to enhance $L E C l$ expression in non-embryonic tissues.

Because pga37 roots showed a phenotype similar to

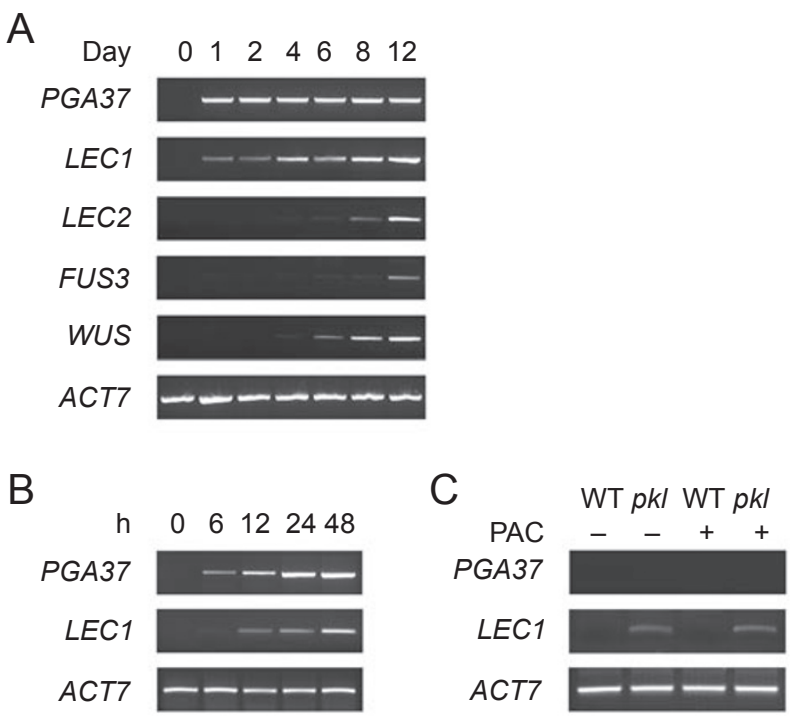

Figure 4 PGA37-regulated expression of LEC1. (A) RT-PCR analysis of PGA37, LEC1, LEC2, FUS3, and WUS expression in pga37 root explants. Root explants cultured on CIM medium (containing 2,4-D; see Materials and Methods) for 3 days were transferred onto SCM. Root explants collected at the time points indicated on the top of the panel were used for the preparation of total RNA. RT-PCR was carried out as described in Figure 3A. PCR was cycled 25 times for PGA37 and ACT7, and 30 times for other genes. (B) RT-PCR analysis of the LEC1 expression in pga37 seedlings. Two-week-old seedlings germinated and grown on MS medium were treated with $10 \mu \mathrm{M}$ estradiol for different times (hours; as indicated on the top of the panel). Expression of $L E C 2$ and WUS was not detected under the assay conditions. (C) Expression of PGA37 and LEC1 in WT and pkl mutant plants. Two-week-old seedlings were germinated and grown on MS medium with (+) or without (-) $20 \mathrm{nM}$ paclobutrazol (PAC). PAC is an inhibitor of gibberellin biosynthesis, which increases the phenotype penetrance of $p k l$ and val1 mutants $[9,12]$. Total RNA was used for RT-PCR analysis as described in Figure 3. RNA prepared from pga37 seedlings treated with estradiol was used as a positive control for the analysis of the PGA37 expression (not shown). 


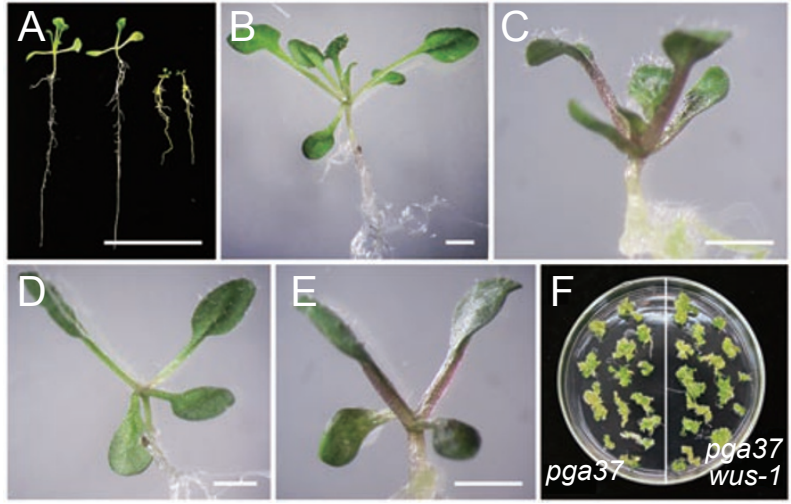

Figure 5 PGA37 functions independently from WUS. (A) Twoweek-old seedlings germinated and grown on MS medium in the presence or the absence of $10 \mu \mathrm{M}$ estradiol. Note that the pga37 wus- 1 double mutant shows a phenotype similar to that of pga37 in the presence of estradiol. Double mutant plants were smaller than WT plants and produced green roots. From left to right: pga37 on MS medium, pga37 wus-1 on MS medium, pga37 germinated on estradiol-containing medium, and pga37 wus-1 germinated on estradiol-containing medium. (B-E) Enlarged views of 2-week-old seedlings under different conditions. (B) A pga37 seedling germinated and grown on MS medium. (C) A pga37 seedling germinated and grown on MS medium containing $10 \mu \mathrm{M}$ estradiol. (D) A pga37 wus-1 seedling germinated and grown on MS medium. (E) A pga37 wus-1 seedling germinated and grown on MS medium containing $10 \mu \mathrm{M}$ estradiol. (F) Root explants derived from pga37 (left) and pga37 wus-1 double mutant (right) plants were cultured as described in panel (A) (pretreated with 2,4-D for 3 days). Bar, $2 \mathrm{~cm}(\mathrm{~A})$ and 2 $\mathrm{mm}$ (others).

that of $p k l$, which produced green roots and had an elevated $L E C 1$ expression level $[9,10]$, it is possible that MYB 118 expression might be elevated in $p k l$ as well. However, MYB 118 expression was not detectable under various conditions in $p k l$, whereas $L E C l$ expression was readily detected as reported previously (Figure 4C) [10], suggesting that MYB118 is not directly regulated by $P K L$.

\section{PGA37 acts independently from WUS}

The pga37 mutant shares some similarities with the pga6 mutant with respect to their capacity to promote vegetative-to-embryonic transition. To investigate possible interactions between WUS/PGA6 and PGA37, we constructed a pga37 wus-1 double mutant by genetic crosses of these two mutants. wus- 1 has been previously shown as a null mutation [24]. In the double mutant, $P G A 37$ expression was inducible by estradiol in the wus-1 loss-of-function mutant background. When germinated and grown in the presence of estradiol, pga37 wus1 showed a pga37-like phenotype, with characteristics of dark-green leaves, light green roots and growth inhibition (Figure 5A). Notably, developmental defects in the shoot apical meristem in wus-1 could not be rescued by $P G A 37$ overexpression (Figure 5B-5E). To test if somatic embryo formation promoted by $P G A 37$ is dependent on WUS, pga37 wus-1 root explants were cultured on a callus induction medium (CIM) and then transferred onto SCM (see Materials and Methods). Root explants derived from both pga37 wus-1 and pga37 plants were able to efficiently form somatic embryos (Figure 5F), suggesting that $W U S$ is not required for $P G A 37$ function in promoting somatic embryo formation.

\section{Functional characterization of MYB115, a homologous} gene of PGA37

PGA37 encodes a R2R3-type MYB transcription factor (MYB118) with unknown function [30, 31]. PGA37/ MYB118 belongs to a small subgroup of six members, classified on the basis of the relatively conserved carboxyl terminal sequences outside the MYB domain [30, 32]. In this family, MYB115 (At5g40360) is most closely related to MYB118 [30]. These two proteins share approximately $32 \%$ identity ( $45 \%$ similarity) in the 150 -amino acid segment located at the carboxyl terminal half. To functionally characterize $M Y B 115$, an estradiol-inducible expression construct pER10-MYB115 was generated, which was stably transformed into WT plants. Expression of the MYB 115 transgene in transgenic plants was highly inducible by estradiol (Figure 6A).

When germinated in the presence of inducer, growth and development of pER10-MYB115 transgenic plants were significantly retarded, and the phenotypic strength was dependent on the transgene expression level (Figure 6A-6C). Overexpression of MYB115 caused a complete arrest of plant growth and development and the transformation of the plantlet into callus-like structures (Figure 6C), a phenotype similar to that of pER10-MYB 118 (see Figure 2G). In root explants derived from pER10MYB 115 transgenic plants, somatic embryos could be efficiently formed in an inducer-dependent manner (Figure 6D-6G). Similar to that observed in pga37 and pER10$M Y B 118$ transgenic plants, expression of $L E C 1$, but not of $L E C 2$ and WUS, was increased in pER10-MYB115 transgenic plants treated with the inducer for 1-2 days (data not shown). The results suggest that the structurally similar MYB118/PGA37 and MYB115 genes have similar functions.

A RT-PCR analysis showed that MYB 115 was expressed in flowers and siliques, but its expression was not detectable in other tissues (Figure $6 \mathrm{H}$ ). The expression pattern is similar to that of $P G A 37 / M Y B 118$ but in a more extended domain (see Figure 3), suggesting that 
A

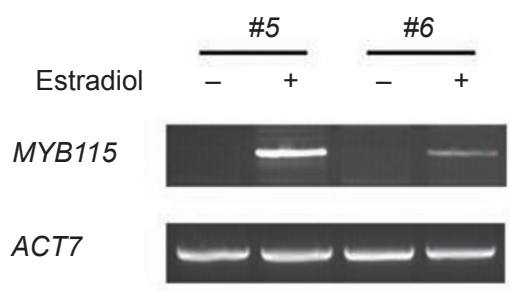

B

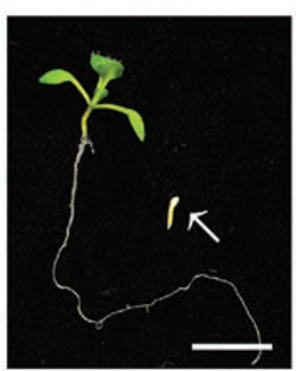

\#6

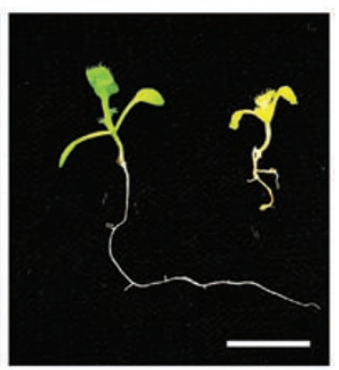

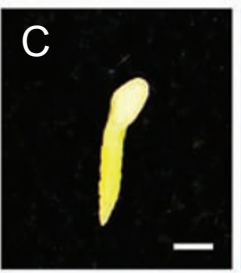
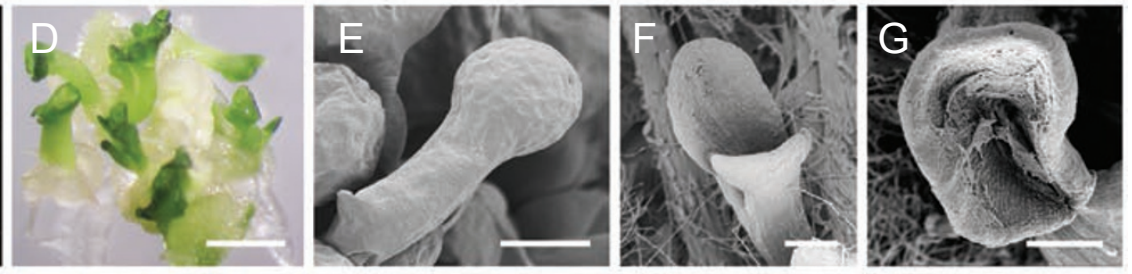

$\mathrm{H}$

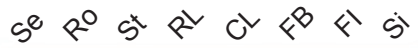

I

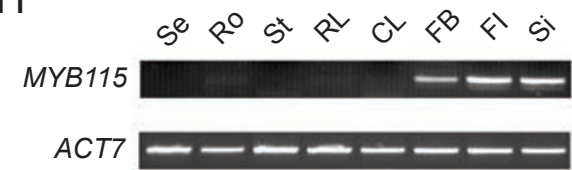

PGA37/MYB118

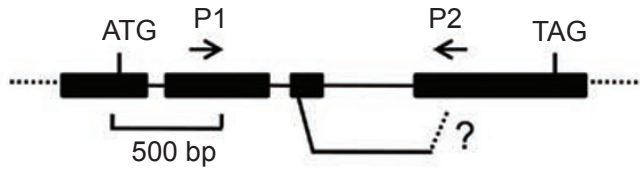

$J$
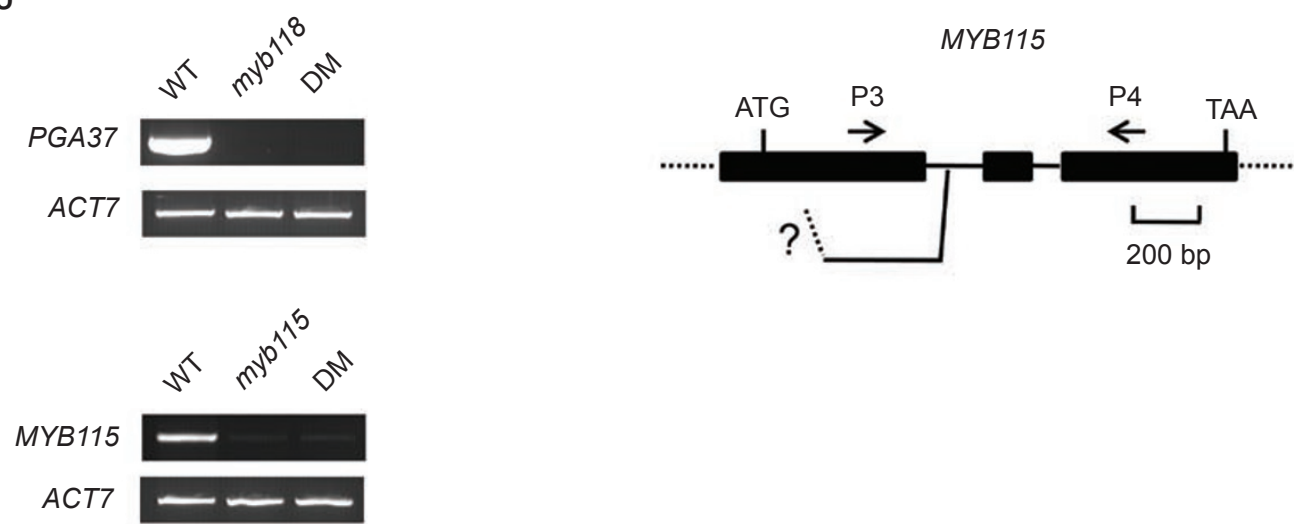

Figure 6 MYB115 functions similarly as PGA37/MYB118. (A) Expression of MYB115 in pER10-MYB115 transgenic plants. Two-week-old pER10-MYB115 transgenic seedlings germinated and grown on MS medium were treated with (+) or without (-) $10 \mu \mathrm{M}$ estradiol for $16 \mathrm{~h}$. Total RNA used for RT-PCR analysis as described in Figure 3A. The PCR was cycled for 25 times. Two transgenic lines (indicated on the top of the panel) with different MYB115 expression levels are shown. (B) Two-week-old pER10-MYB115 transgenic seedlings germinated and grown on MS medium supplemented with (right in each panel) or without (left in each panel) $10 \mu \mathrm{M}$ estradiol. Two transgenic lines (\#5 and \#6) are shown. (C) An enlarged view of a plantlet shown in panel (B) marked by an arrow. (D) Germinating somatic embryos from pER10-MYB115 root explants cultured on SCM for 4 weeks. (E-G) Scanning electron microscopy of somatic embryos derived from pER10-MYB115 root explants cultured on SCM for 4 weeks. (E) A somatic embryo at the globular stage. (F) A somatic embryo at the cotyledon stage. (G) A somatic embryo with fused cotyledons. (H) Expression pattern of MYB115 analyzed by RT-PCR. Se: seedling; Ro: root; St: stem; RL: rosette leaf; CL: cauline leaf; FB: flower bud; FI: flower; Si: silique. (I) Schematic maps of the myb118 (top) and myb115 (bottom) mutant genomes. A T-DNA was inserted in exon 3 of PGA37/MYB118, and a T-DNA was inserted in intron 1 of MYB115. Filled boxed and lines denote exons and introns, respectively. Dashed lines indicate non-transcribed sequences. Arrows above exons show the positions and orientation of primers used for RT-PCR analyses shown in panel (J). (J) Expression of PGA37/ MYB118 (top) and MYB115 (bottom) in the myb118 myb115 double mutant. Total RNA prepared from immature siliques of WT, single, and double mutant plants was used for RT-PCR analysis. DM: the myb118 myb115 double mutant. Bar, $0.5 \mathrm{~cm}(\mathrm{~B})$, $0.5 \mathrm{~mm}$ (C and D), $50 \mu \mathrm{m}(\mathrm{E})$, and $200 \mu \mathrm{m}$ ( $F$ and $\mathrm{G})$. 
these two genes have similar but distinctive functions during plant growth and development. To further investigate the function of $P G A 37 / M Y B 118$ and MYB115 in plant growth and development, we identified two T-DNA insertion lines SALK 111812 and SALK 044168 [33]. These two lines contained T-DNA insertions in exon 3 of $P G A 37 / M F Y B 118$ and intron 1 of $M Y B 115$, respectively (Figure 61). Expression of $P G A 37 / M Y B 118$ was not detected in siliques of myb118 and myb118 myb115 plants (Figure $6 \mathrm{~J}$ ), suggesting that the T-DNA insertion likely caused a null mutation. However, reduced expression of MYB 115 was detected in myb115 and myb118 myb115 siliques (Figure 6J), which presumably resulted from cryptic splicing of myb115 pre-mRNAs. Homozygous myb118 and myb115 plants were phenotypically indistinguishable from WT plants. In addition, no abnormalities were found in the myb118 myb115 double mutant at different developmental stages. When germinated and grown in the presence of auxin, cytokinin, abscisic acid or sucrose, the myb118 myb115 double mutant showed a normal response to these compounds as WT.

\section{Discussion}

In this study, we show that overexpression of $P G A 37 /$ MYB 118 and MYB115, two homologous Arabidopsis genes, efficiently induces the formation of somatic embryos from root explants, resulting in elevated expression levels of the embryo-specific gene LEC1. Moreover, overexpression of $P G A 37 / M Y B 118$ and MYB 115 also causes the accumulation of lipids in vegetative tissues, the formation of green roots, and the disruption of postgermination morphogenesis. These observations suggest that $P G A 37 / M Y B 118$ and MYB115 are able to promote reprogramming of cell fate, acting as positive regulators of vegetative-to-embryonic transition.

The pga37 mutant shows phenotypic similarities with pga6 and transgenic plants overexpressing $B B M[15$, $21]$. The most striking phenotype of these mutants and transgenic plants is their capacity to form somatic embryos from vegetative tissues. Several lines of evidence suggest that PGA37 may act independently from WUS/ $P G A 6$. First, WUS expression is restricted to the organizing center during embryogenesis [24]. However, PGA37 is expressed in a more expanded domain, including both the embryo and the endosperm, similar to the expression pattern of $B B M$ [15]. Although a non-cell-autonomous regulatory mechanism could not be excluded, it is unlikely that $W U S$ directly targets $P G A 37$. Second, overexpression of $P G A 37$ is unable to rescue the shoot apical meristem defects in wus-1, thus disfavoring a model that $P G A 37$ acts downstream of WUS. Third, WUS represses
[21], whereas $P G A 37$ promotes, $L E C 1$ expression in root explants or embryogenic cells. Moreover, in pga6 root explants, $L E C l$ expression was found to be induced around 14 days after cultured on SCM [21]. By contrast, LEC1 expression is detectable in pER10-PGA37 seedlings treated with estradiol for $12 \mathrm{~h}$. These observations lead to the speculation that LEC1 expression observed in pga6 may represent a characteristic of embryogenic calli or somatic embryos. A similar observation was recently made by finding that $L E C 2$-induced expression of genes encoding the seed proteins oleosin and storage proteins is prior to that of $L E C 1$ and FUS3 [4]. In contrast to that of WUS/PGA6 and LEC2, PGA37 may act upstream of $L E C 1$ to regulate vegetative-to-embryonic transition. Therefore, these different regulatory modes do not support the hypothesis that $W U S$ and $P G A 37$ act in a linear pathway. Instead, different mechanisms may be deployed by $W U S / P G A 6$ and $P G A 37$ to regulate vegetative-toembryonic transition.

Compared to the previously characterized genetic loci involved in the regulation of vegetative-to-embryonic transition such as WUS/PGA6 and BBM, PGA37 employs a unique regulatory mechanism. The $P G A 37$ activity is independent of WUS in the regulation of vegetative-toembryonic transition. Moreover, $P G A 37 / M Y B 118$ is capable of enhancing $L E C 1$ expression, but does not appear to be regulated by $P K L$. Whereas $P K L$ has been shown to repress $L E C 1$ expression [10], the expression of $P K L$ itself is, in turn, positively regulated by $L E C 1$, suggesting the involvement of a feedback regulatory mechanism (J $\mathrm{Mu}$ and $\mathrm{J}$ Zuo, unpublished data). Similarly, expression of LEC1, but not of $P G A 37 / M Y B 118$ and MYB115, is remarkably elevated in the vall val2 double mutant [12]. Notably, overexpression of PGA37/MYB118 and MYB115 induces the expression of $L E C 1$ but not $L E C 2$, consistent with the observation made in previous studies that these two loci may act independently in the control of embryo development and seed maturation [34]. Collectively, these results suggest that $P G A 37 / M Y B 118$ and MYB115 may regulate $L E C 1$ expression in a $P K L$-independent manner. $L E C 1$ is a key regulator of embryogenesis and seed maturation [5-7, 35]. Overexpression of LECl is sufficient to induce vegetative-to-embryonic transition [7], indicating a critical role of $L E C 1$ in the initiation and/or maintenance of embryonic cell identity. Therefore, the promotion of vegetative-to-embryonic transition by $P G A 37 / M Y B 118$ and MYB 115 should be attributed, at least partly, to an elevated $L E C 1$ expression level.

$P G A 37 / M Y B 118$ is preferentially expressed in embryos and endosperm, implying a possible role of the MYB transcription factor in embryogenesis and seed development. This notion is supported by the phenotype 
of the gain-of-function mutants and of transgenic plants overexpressing $P G A 37$. However, neither myb118 nor the myb118 myb115 double mutant displays any developmental defects under our assay conditions. The lack of an apparent phenotype may be caused by functional redundancy of this small class of MYB transcription factors and, in particular, owing to the residual expression of MYB115 in the double mutant. Alternatively, genetic redundancy may be present between the MYB118/MYB115 family and other transcription factor genes. Despite the lack of an apparent phenotype in myb118 and myb118 myb115 mutants, overexpression of these two genes causes strong inhibitory effects on plant growth and development and promotes the formation of embryonic calli on seedlings, suggesting that these two functionally redundant genes likely play a role in embryo or seed development.

In summary, we have identified and characterized PGA37/MYB118 and MYB115 that are capable of inducing vegetative-to-embryonic transition and the formation of somatic embryos. Because PGA37/MYB118 acts independently from $W U S$, the finding presented in this study thus reveals a new regulatory mechanism of somatic embryogenesis in Arabidopsis, which should have substantial impacts on both plant biotechnology and our understanding of this fundamental question in plant biology.

\section{Materials and Methods}

Plant material, growth conditions, and plant transformation The Arabidopsis thaliana ecotypes Col-0, WS, and Landsberg erecta (Ler) were used in this study. Screening of pga mutants has been described in detail previously [21, 22]. The myb118 (SALK_111812; Col-0), myb115 (SALK_044168; Col-0), and wus-1 (CS15; Ler) mutants were obtained from the Arabidopsis Biological Resource Center (ABRC, Ohio State University, Columbus, OH). The pkl-1 mutant (Col-0) [9] was kindly provided by J Ogas.

Plants were grown at $22{ }^{\circ} \mathrm{C}$ under a 16-h-light/8-h-dark cycle in soil or on agar plates containing 1/2 MS medium (Sigma Hong Kong and China), $2 \%$ sucrose, and $0.8 \%$ agar supplemented with or without the inducer $17-\beta$-estradiol. Treatment of plants with $17-\beta$-estradiol was carried out as described previously [20]. Transformation of root explants was carried out using Agrobacterium tumefaciens (the ABI strain) as described previously [21]. Unless indicated otherwise, root explants were cultured on a CIM $(1 \times$ B5 salts, 2\% glucose, $0.5 \mathrm{~g} / \mathrm{L}$ MES, $0.5 \mathrm{mg} / \mathrm{L} 2,4-\mathrm{D}, 0.05 \mathrm{mg} / \mathrm{L}$ kinetin and $0.25 \%$ phytagel, $\mathrm{pH} 5.7$ ) for 3 days, and subsequently transferred onto a SCM ( $1 \times$ MS salts, $1 \%$ sucrose, $0.5 \mathrm{~g} / \mathrm{L} \mathrm{MES,}$ $0.15 \mathrm{mg} / \mathrm{L}$ IAA, $10 \mu \mathrm{M} 17-\beta$-estradiol, and $0.25 \%$ phytagel, $\mathrm{pH} 5.7$ ) for 3-5 weeks. In planta transformation was done by the flowerdip method [36] using the Agrobacterium GV3101 strain.

Staining of seedlings with Fat Red 7B was carried out according to [9]. Scanning electron microscopy was performed as de- scribed previously [37].

\section{Molecular manipulations}

Molecular cloning was performed according to standard methods [38]. Genomic sequences flanking the $O^{L e x A}-46$ promoter in the pga37 genome were identified by inverse PCR [29]. A PGA37/ MYB 118 cDNA clone and a MYB115 (AT5g40360) genomic clone were obtained by PCR using PWO DNA polymerase (Roche Diagnostics Hong Kong and China) and appropriate primer pairs (all primers used in this study are listed in Supplementary information, Table S1). The PCR fragments were cloned into the AscI (PGA37/ MYB118) or XhoI (MYB115) and SpeI sites of the estradiolinducible vector $\mathrm{pER} 10$ [20].

To generate the PGA37::GUS reporter construct, a $2.5-\mathrm{kb}$ genomic fragment, which spanned nucleotides -2535 to +36 with respect to the putative translation start codon, was amplified from Col-0 genomic DNA using KOD-Plus DNA polymerase (Toyobo, Osaka, Japan) and primers PGA37PF and PGA37PB. The PCR fragment, containing the coding sequence for the first 12 amino acid residues of PGA37, was in-frame fused to the GUS coding sequence in pBI101.1 using the $X b a \mathrm{I}$ and $B a m H I$ sites. All constructs were verified by extensive restriction digests and DNA sequencing.

Analysis of gene expression by RT-PCR was performed essentially as described previously [22].

\section{Analysis of GUS activity}

GUS staining was performed as described [39]. Histochemical staining of siliques was carried out as described [40] with minor modifications. Briefly, siliques were slit twice longitudinally and incubated in $90 \%$ acetone for $20 \mathrm{~min}$ at room temperature. After extensive wash, the sample was incubated in a GUS assay buffer (50 $\mathrm{mM}$ phosphate buffer [pH 7.2], 0.1\% Triton X-100, 2 mM X-Gluc, $2 \mathrm{mM}$ potassium ferrocyanide and $2 \mathrm{mM}$ potassium ferricyanide) for about $6 \mathrm{~h}$ at $37^{\circ} \mathrm{C}$. After staining, siliques were cleared in ethanol-acetic acid and then mounted in Hoyer's medium. Dissected ovules were observed and photographed under an Olympus BX51 microscope equipped with Nomarski optics and a cooled KX85 CCD camera.

\section{Acknowledgments}

We are grateful to Dr Ying Lan and Mr Bin Xu (State Key Laboratory of Plant Genomics, Institute of Genetics and Developmental Biology, Chinese Academy of Sciences) for their technical assistance in scanning electronic microscopy. We would like to thank Dr Joe Ogas (Purdue University) and the Arabidopsis Biological Resource Center for mutant seeds. This work was supported by grants from the Ministry of Science and Technology of China (grant numbers: 2007CB948203 and 2006CB101601), National Natural Science Foundation of China (grant numbers: 30600047 and 30221002), and the Chinese Academy of Sciences (KSCX2-YW-N-015). Work done at the Rockefeller University was supported by a grant from DuPont.

\section{References}

1 Zimmerman JL. Somatic embryogenesis: a model for early 
development in higher plants. Plant Cell 1993; 5:1411-1423.

2 Mordhorst AP, Toonen MAJ, de Vries SC. Plant embryogenesis. Crit Rev Plant Sci 1997; 16:535-576.

3 Sugiyama M. Organogenesis in vitro. Curr Opin Plant Biol 1999; 2:61-64.

4 Stone SL, Braybrook SA, Paula SL, et al. Arabidopsis LEAFY COTYLEDON2 induces maturation traits and auxin activity: implications for somatic embryogenesis. Proc Natl Acad Sci USA 2008; 105:3151-3156.

5 Meinke DW, Franzmann LH, Nickle TC, Yeung EC. Leafy cotyledon mutants of Arabidopsis. Plant Cell 1994; 6:10491064.

6 West MAL, Yee KM, Danao J, et al. LEAFY COTYLEDON1 is an essential regulator of late embryogenesis and cotyledon identity in Arabidopsis. Plant Cell 1994; 6:1731-1745.

7 Lotan T, Ohto M, Yee KM, et al. Arabidopsis LEAFY COTYLEDON1 is sufficient to induce embryo development in vegetative cells. Cell 1998; 93:1195-1205.

8 Stone SL, Kwong LW, Yee KM, et al. LEAFY COTYLEDON2 encodes a B3 domain transcription factor that induces embryo development. Proc Natl Acad Sci USA 2001; 98:11806-11811.

9 Ogas J, Cheng JC, Sung ZR, Somerville C. Cellular differentiation regulated by gibberellin in the Arabidopsis thaliana pickle mutant. Science 1997; 277:91-94.

10 Ogas J, Kaufmann S, Henderson J, Somerville C. PICKLE is a CHD3 chromatin-remodeling factor that regulates the transition from embryonic to vegetative development in Arabidopsis. Proc Natl Acad Sci USA 1999; 96:13839-13844.

11 Henderson JT, Li HC, Rider SD, et al. PICKLE acts throughout the plant to repress expression of embryonic traits and may play a role in gibberellin-dependent responses. Plant Physiol 2004; 134:995-1005.

12 Suzuki M, Wang HHY, McCarty DR. Repression of the $L E A F Y$ COTYLEDON 1/B3 regulatory network in plant embryo development by VP1/ABSCISIC ACID INSENSITIVE 3-LIKE B3 genes. Plant Physiol 2007; 143:902-911.

13 Schmidt ED, Guzzo F, Toonen MA, de Vries SC. A leucinerich repeat containing receptor-like kinase marks somatic plant cells competent to form embryos. Development 1997; 124:2049-2062.

14 Hecht V, Vielle-Calzada JP, Hartog MV, et al. The Arabidopsis SOMATIC EMBRYOGENESIS RECEPTOR KINASE 1 gene is expressed in developing ovules and embryos and enhances embryogenic competence in culture. Plant Physiol 2001; 127:803-816.

15 Boutilier K, Offringa R, Sharma VK, et al. Ectopic expression of $B A B Y B O O M$ triggers a conversion from vegetative to embryonic growth. Plant Cell 2002; 14:1737-1749.

16 Harding EW, Tang W, Nichols KW, Fernandez DE, Perry SE. Expression and maintenance of embryogenic potential is enhanced through constitutive expression of AGAMOUS-Like 15. Plant Physiol 2003; 133:653-663.

17 Thakare D, Tang W, Hill K, Perry SE. The MADS-domain transcriptional regulator AGAMOUS-Like 15 promotes somatic embryo development in Arabidopsis thaliana and Glycine max. Plant Physiol 2008; 146:1663-1672.

18 Wang H, Caruso LV, Downie AB, Perry SE. The embryo MADS domain protein AGAMOUS-Like 15 directly regulates expression of a gene encoding an enzyme involved in gibber- ellin metabolism. Plant Cell 2004; 16:1206-1219.

19 Gazzarrini S, Tsuchiya Y, Lumba S, Okamoto M, McCourt P. The transcription factor FUSCA3 controls developmental timing in Arabidopsis through the hormones gibberellin and abscisic acid. Dev Cell 2004; 7:373-385.

20 Zuo J, Niu QW, Chua NH. An estrogen receptor-based transactivator XVE mediates highly inducible gene expression in transgenic plants. Plant J 2000; 24:265-273.

21 Zuo J, Niu QW, Frugis G, Chua NH. The WUSCHEL gene promotes vegetative-to-embryonic transition in Arabidopsis. Plant J 2002; 30:349-359.

22 Sun J, Niu QW, Tarkowski P, et al. The Arabidopsis AtIPT8/ $P G A 22$ gene encodes an isopentenyl transferase that is involved in de novo cytokinin biosynthesis. Plant Physiol 2003; 131:167-176.

23 Laux T, Mayer KF, Berger J, Jurgens G. The WUSCHEL gene is required for shoot and floral meristem integrity in Arabidopsis. Development 1996; 122:87-96.

24 Mayer KFX, Schoof H, Haecker A, et al. Role of WUSCHEL in regulating stem cell fate in the Arabidopsis shoot meristem. Cell 1998; 95:805-815.

25 Tucker MR, Laux T. Connecting the paths in plant stem cell regulation. Trends Cell Biol 2007; 17:403-410.

26 Gallois JL, Nora FR, Mizukami Y, Sablowski R. WUSCHEL induces shoot stem cell activity and developmental plasticity in the root meristem. Genes Dev 2004; 18:375-380.

27 Xu YY, Wang XM, Li J, et al. Activation of the WUS gene induces ectopic initiation of floral meristems on mature stem surface in Arabidopsis thaliana. Plant Mol Biol 2005; 57:773784.

28 Murashige T, Skoog F. A revised medium for rapid growth and bioassays with tobacco tissue culture. Physiol Plant 1962; 15:473-497.

29 Ochman H, Gerber AS, Hartl DL. Genetic applications of an inverse polymerase chain reaction. Genetics 1988; 120:621623.

30 Stracke R, Werber M, Weisshaar B. The R2R3-MYB gene family in Arabidopsis thaliana. Curr Opin Plant Biol 2001; 4:447-456.

31 Yanhui C, Xiaoyuan Y, Kun H, et al. The MYB transcription factor superfamily of Arabidopsis: expression analysis and phylogenetic comparison with the rice MYB family. Plant Mol Biol 2006; 60:107-124.

32 Kranz HD, Denekamp M, Greco R, et al. Towards functional characterisation of the members of the R2R3-MYB gene family from Arabidopsis thaliana. Plant J 1998; 16:263-276.

33 Alonso JM, Stepanova AN, Leisse TJ, et al. Genome-wide insertional mutagenesis of Arabidopsis thaliana. Science 2003; 301:653-657.

34 To A, Valon C, Savino G, et al. A network of local and redundant gene regulation governs Arabidopsis seed maturation. Plant Cell 2006; 18:1642-1651.

35 Parcy F, Valon C, Kohara A, Misera S, Giraudat J. The $A B$ SCISIC ACID-INSENSITIVE3, FUSCA3, and LEAFY COTY$L E D O N 1$ loci act in concert to control multiple aspects of Arabidopsis seed development. Plant Cell 1997; 9:1265-1277.

36 Bechtold N, Ellis J, Pelletier G. In planta Agrobacteriummediated gene transfer by infiltration of adult Arabidopsis thaliana plants. C R Acad Sci Ser III Sci Vie 1993; 316:1194- 
1199.

37 Feng H, Chen Q, Feng J, et al. Functional characterization of the Arabidopsis eukaryotic translation initiation factor 5A-2 that plays a crucial role in plant growth and development by regulating cell division, cell growth, and cell death. Plant Physiol 2007; 144:1531-1545.

38 Sambrook J, Russell DW. Molecular Cloning: A Laboratory Manual. Cold Spring Harbor, NY: Cold Spring Harbor Labora- tory Press, 2001.

39 Jefferson RA, Kavanagh TA, Bevan MW. GUS fusions: betaglucuronidase as a sensitive and versatile gene fusion marker in higher plants. EMBO J 1987; 6:3901-3907.

40 Stangeland B, Salehian Z. An improved clearing method for GUS assay in Arabidopsis endosperm and seeds. Plant Mol Biol Rep 2002; 20:107-114.

(Supplementary information is linked to the online version of the paper on the Cell Research website.) 